Cell Research(1995),5,9-24

\title{
Laser scanning fluorescence microscopic measurement of the movement of cleaving egg surface of Rana Amurensis ${ }^{1,2}$
}

\author{
GU Guoyan ( Formerly KU Kuoyen), Chengtang \\ XU, Konghua ZHANG, QIRONG GAO. \\ Shanghai Institute of Cell Biology, Chinese Academy of \\ Sciences, Shanghai 200031, China.
}

\begin{abstract}
By laser scanning fluorescence microscopy for quantitative measurement of fluorescence intensity changes on egg surface stained with fluorescein isothiocvanate during cleavage furrow extending forward, it was found that in area of presumptive cleavage furrow the scanning curve became $\mathrm{V}$ shape, indicating dark stripe appeared in that place. Then the fluorescence intensity increased at the place where the bottom of $\bigvee$ shape had located, and the scanning curve turned to $\bigwedge$ shape, indicating single stripe was formed. While enhanced fluorescence appeared on the borders of $\bigwedge$ shape, an $M$ shape curve was found, showing double stripe occurred. During the distance between two borders of $\mathrm{M}$ shape increasing from $50 \mu \mathrm{m}$ to $100 \mu \mathrm{m}$, a fluorescence peak came to sight in the middle of the $\mathrm{M}$ shape, which being the cleavge furrow bottom. The two lateral sides of furrow bottom with decreasing fluorescence were nascent membrane. At that time the curve became $\mathrm{W}$ shape. By the sides of cleavage furrow the the stress folds became conspicous after double stripe stage, showing the stretching of the egg surface being increased. With our[31, 33] and others[32] reports that polylysine could induce the appearance of nascent membrane and phytohemagglutinins could decrease or prevent the appearance of nascent membrane, we believed the idea of Schroeder[25]
\end{abstract}

1. Dedicated to Prefessor Zhen YAO on the occasion of his 80th birthday.

2. This work was supported by the director's foundation of Shanghai Institute of Cell Biology 
that increasing mechanical stress could initiate nascent membrane formation and thought that the stress lay to the outsides of cleavage furrow.

Key words: cleavage, egg surface movement, initiating nascent membrane formation, laser scanning fluorescence microscopy, Rana Amurensis.

\section{INTRODUCTION}

Previous studies have indicated that cleavage furrow of amphibian egg succeeded in dividing on an excised piece of egg surface including an area of a furrow tip[1] and on a graft including a furrow on host egg[2]. These results implied that physical mechanism of cleavage procession is located within cell surface. In recent years Mabuchi et al[3] isolated early stage cleavage furrows from newt eggs, it could contract when energy was supplied. They postulated that it was resulted from the contraction of actin in cortex directly or indirectly $[4,5]$ bringing along the plasma membrane and glycocalyx. The cleavage furrow formation of amphibian egg went through dark[6], single, double stripe and groove stages[7, 8]. During these stages whether there is only one form or several forms of movements? Is the mechanism of formation and extension of cleavage furrow alike? these are problems important to elucidate the biomechanics of the movement of cleaving egg suface[9] and the simulation of the procession of cytokinesis[10]. It was known the fluorescence intensity of fluorescein isothiocvanate stained egg surface changed with the formation of cleavage furrow[6], so we used laser scanning fluorescence microscopy to measure quantitatively the changes of fluorescence intensity and found that there were several kinds of movement during cleavage and the formation and extension of cleavage furrow were much alike. Based on the movement of egg surface the initiation of nascent membrane formation is discussed.

\section{MATERIAL and METHODS}

\section{Egg of Rana amurensis}

A female Rana amurensis was injected with homogenate of pituitary glands of the same species to induce ovulation. Stripped eggs were put into petri dishes and sperm suspension was added to fertilize the eggs. Fertilized eggs were stripped by treatment with mercaptoethanol at $\mathrm{pH}$ 8.5. Eggs enclosed in fertilization membrane were stained with $\mathrm{Ca}^{2+}$ and $\mathrm{Mg}^{2+}$ free Barth solution containing $1 \mathrm{mg} / \mathrm{ml}$ fluorescein isothiocyanate (FITC, isomer I, Sigma) at $\mathrm{pH} 10$ for about $10 \mathrm{~min}$, at room temperature. Fertilization membrane was removed with forceps. The naked egg either in living state or fixed briefly in 10\% formalin was transferred with their black animal hemisphere upward 
$\mathrm{Ku} \mathrm{KY}$ et al.

into a small chamber containing Barth solution. The chamber and a cover glass were sealed together with silicon grease.

\section{Laser scanning fluorescence microscope}

The $488 \mathrm{~nm}$ line of Argon laser (Naniing Electronic Tube Corp. Model 360) was entered into fluorescence microscope (Leitz Ortholux 2) as shown in Fig 1. The objective lens of microscope was focused on surface of egg in small chamber lain on $360^{\circ}$ rotatory plate fixed on microscopic stage which was connected with stepping motor. The speed and direction of motion of the microscopic stage was controlled by computer(PC/XT) through a driving unit (Lang-electronik MC-12JS). The fluorescence intensity emitted from egg surface was accepted by photoelectron tube (EMI 9789 QB) and recorded in computer which operated on-1ine with the motion of microscopic stage in X-Y direction. The records stored in computer were drawn on a chart with graph plotter(FWX-4675).

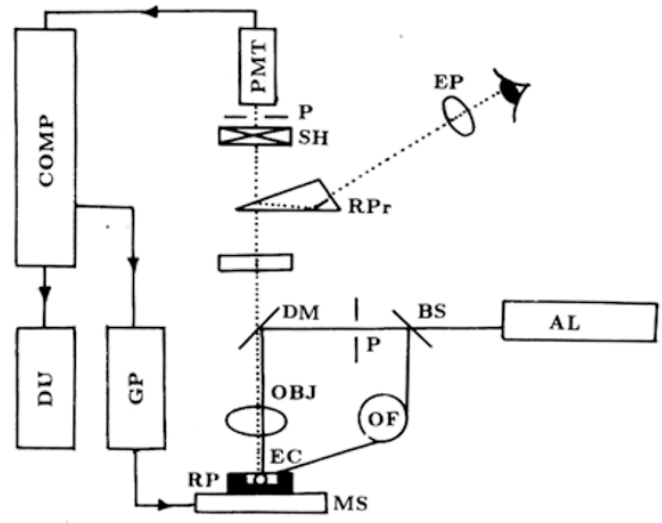

Fig 1. Diagram of the apparatus of laser scanning fluorescence microscope AL: argon laser. BS: beam splitter. COMP:computer. DM:dichroic mirror. DU: driving unit. EC: egg chamber. EP: eyepiece. GP: graph plotter. MS: microscopic stage movable in X-Y direction. OF: optical fibers. OBJ: objective. P: pinhole. PMT: photomultiplier. RP: rotatory plate. RPR: reflective prism. SF: suppression filter. SH: shutter.

....., fluorescence light path.

, laser light path.

\section{Scanning fluorescence microscopy}

The egg was watched under objective lens, $6.3 \mathrm{x}$, with the help of diffusion light from laser through glass fibers. Just after the onset of cleavage the egg was rotated to make the first cleavage furrow perpendicular to the direction of scan line and the objective lens was changed to $25 \mathrm{x}$. The beam was focused on the cleavage furrow at which the diameter of light spot was $6.8 \mu \mathrm{m}$. First scan line was drawn from left side of egg through the front of furrow tip to right side of egg. The following scan lines were drawn in same direction in parallel with and $100 \mu \mathrm{m}$ apart from the above. On a formalin fixed egg 10 scan lines were obtained, On living cleaving egg 5 scan lines as above were done in $2 \mathrm{~min}$, then repeatedly scanned at the "same places" for 9 times within $18 \mathrm{~min}$.

\section{RESUITS}

\section{Fluorescence scanning curves on formalin fixed eggs}

In order to illustrate the quantitative correlation between the changes of fluorescence intensity and morphological changes of egg surface during the development of cleavage furrow, it needs to understand the variation or the unevenness of fluorescence staining on egg surface. We measured the fluorescence intensity within $20 \mu \mathrm{m}$ width on both sides of central point between border lines of egg and c leavge 
Movement of cleaving frog egg surface

furrow (abbreviated as EB-CFB point) in scanning curves (as Fig 2B) of formalin fixed eggs (as Fig 2A) and calculated the evenness of fluorescence staining according to following formula:

the difference between the maximum and minimum light intensity in $40 \mu \mathrm{m}$ width $\times 100 \%$

the light intensity on EB-CFB point

The fluorescence of $40 \mu \mathrm{m}$ width of $99 \mathrm{~EB}-\mathrm{CFB}$ points of 6 eggs were varied from $1.10 \%$ to $14.68 \%$, with mean $5.45 \%$, and standard deviation, \pm 2.36 . Therefore when the fluorescence intensity within $40 \mu \mathrm{m}$ was $15 \%$ greater than the intensity at its both sides, it was considered as morphological changes being appeared at that place. For examples, in Fig 2B, there showed a peak on the 8th curve that corresponded to the polar body extrusion point in Fig $2 \mathrm{~A}$ and one hollow on the 8th curve and 2 hollows on 10th curves corresponded to the light fluorescence patches on egg surface in Fig $2 \mathrm{~A}$.

The changes of fluorescence intensity at different parts of cleavage furrow and around were as follows. When the laser beam passing the front of cleavage furrow tip (Fig 2A), the light intensity decreased forming a $\mathrm{V}$ shape (Fig 2B, 1st. curve). This represented $V$ stage, corresponding to dark stripe stage of cleavage furrow development[6]. When the beam passing the rear of furrow tip (Fig 2A), the light intensity increased forming a $\Lambda$ shape (Fig 2B, 2nd curve). This was $\Lambda$ stage, equivalent to single stripe stage of furrow development[7, 8]. The small peak on right side of $\Lambda$ shape (Fig 2B) corresponded to stress fold (Fig 2A). In the central part of the 3rd curve close to $\mathrm{M}$ shape was equivalent to double stripe stage[7,8] (Fig 2A) with its width and intensity being both greater than that of $\Lambda$ stage. On the left and right sides of $\mathrm{M}$ curve appeared stress folds and dark stripe respectively. The central part of 4th curve was in $\mathrm{W}$ shape, which being the groove stages[7, 8] (Fig 2A) with its light intensity increasing in stress folds and border lines. In groove a peak appeared between border lines of furrow, this was groove bottom, the location of contractile arc. When the intensity on both sides of furrow bottom was $15 \%$ lower than that of EB-CFB point, it represented the nascent membrane. The 5th curve was quite similar to the 4th. with only slight changes in width and intensity of furrow and the position and number of stress folds as well. The 6th to 10 th curves were much alike to the 5 th to 1 st curves. These results indicated that the changes of fluorescence intensity in cleavage furrow region could represent the morphological changes of celavage furrow on egg surface.

Although cleavage furrow formation went through $\mathrm{V}, \Lambda, \mathrm{M}$ and $\mathrm{W}$ stages, the shapes of whole early cleavage furrow varied in eggs from different individuals of same species. In some cases when furrow length even attained to $40 \%$ of egg diame- 
$\mathrm{Ku} \mathrm{KY}$ et al.


Distance(mm)

Fig 2 A. Fluorescence photo of FITC-stained and formalin-fixed egg during cleavage.

$1-10$, the positions of 1-10th scan lines. The interval between two lines was $100 \mu \mathrm{m}$

B. Fluorescence intensity curves of egg surface in Fig $2 \mathrm{~A}$.

1-10 fluorescence intensity curves corresponding to 1-10th scan lines in Fig 2A.

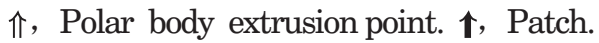

$\uparrow$, Stress fold. $\triangle$, Dark stripe. 
ter the nascent membrane still did not appear (Fig 3A). While in other cases the nascent membrane appeared when the furrow length was not over $30 \%$ of the egg diameter (Fig 3B). However, eggs from one frog all had quite similar early shape of furrow. It seemed that the morphology of cleavage furrow in early development was genetically controlled.
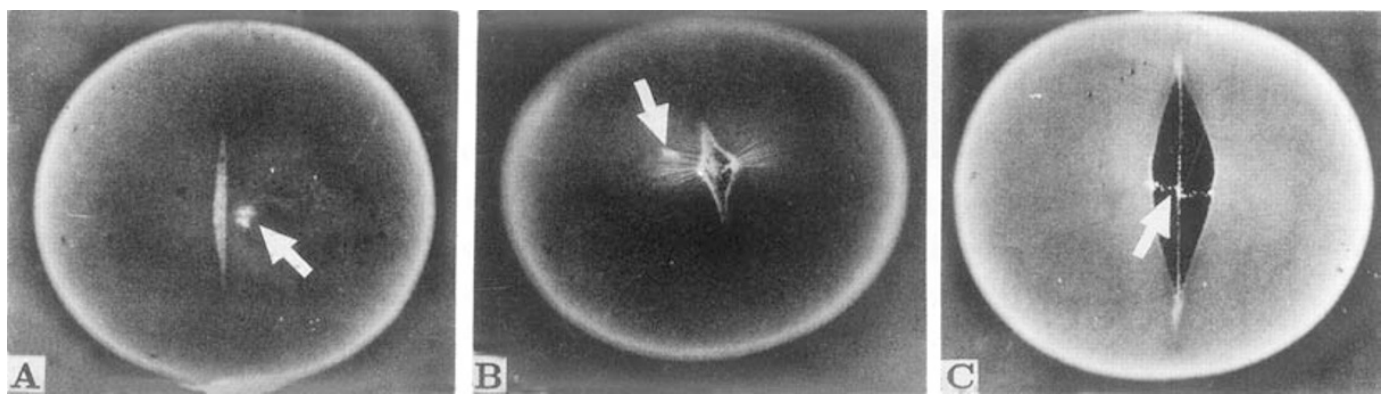

Fig 3. Fluorescence photos of FITC-stained and formalin-fixed eggs during early cleavage.

A. The nascent membrane had not yet appeared when the leagth of cleavage furrow attained $40 \%$ of egg diameter. $\Uparrow$, polar body extrusion point.

B. The nascent membrane had appeared when the length of cleavage furrow attained less than $30 \%$ of egg diameter. In nascent membrane many fluorescence granules could be seen介, polar body extrusion point.

C. The cleavage furrow went through polar body extrusion point which was scattered by the pulling of border lines of cleavage furrow and groove bottom. $\Uparrow$, The scattered polar body extrusion point.

\section{Fluorescence scanning curves on living eggs}

Whether the fluorescence intensity of living eggs would change during continuous scanning of a certain point? We measured the fluorescence intensity at one EB-CFB point at 2 min interval and calculated according to the following formula:

the difference of fluorescence intensity at one EB-CFB point at 2 min interval

the average intensity at one EB-CFB point at 2 min interval

The fluorescence intensity changes of 106 EB-CFB points of 6 eggs between 2 min were varied from $0-4.73 \%$. Therefore when the light intensity changed at one point of $6.8 \mu \mathrm{m}$ diameter in $2 \mathrm{~min}$ was over $5 \%$, it was considered as morphological changes occurred at that place. In Fig 4 , the 5 columns, $\mathrm{C}_{1}-\mathrm{C}_{5}$, represented the fluorescence intensity at 5 difierent places in $100 \mu \mathrm{m}$ apart each of a cleaving egg and the 10 rows, $R_{1}-R_{10}$, represented the changes of fluorescence intensity at these positions for every $2 \mathrm{~min}$. intervals for $20 \mathrm{~min}$. From these curves one might recog- 
nize that Fig 4 represented the development of a short, wide and slowly extended cleavage furrow with early appearing nascent membrane.

\section{A. The movement of cleavage furrow and its around}

a. $V$ stage. In $\mathrm{C}_{1}$ of Fig 4 the central part of $R_{1}-R_{7}$ displayed $V$ shape and varied in width and intensity, which indicated that the dark stripe at furrow tip was unceasingly moving. The duration of the existence of stage varied. In 13 eggs, 4 had existing time over $6 \mathrm{~min}, 1$ over $4 \mathrm{~min}$, and 5 over $2 \mathrm{~min}$. 3 of them did not appear. The reason may be due to the existing time being less than 2 min or the dark stripe being not dark enough to distinguish. The length of dark stripe in furrow tip could exceed $100 \mu \mathrm{zm}$ That meant the curves of two adjacent columns all had dark stripes appearing simultaneously, just like that seen on $R_{1}-R_{2}$ of $C_{1}-C_{2}$ in Fig 4. This phenomenon was only manifested in 4 of 13 eggs and 3 of them manifested when dark stripe existing over $6 \mathrm{~min}$, which indicated that when the length of dark stripe increased, its existing time also prolonged.

b. $\Lambda$ stage. In $\Lambda$ stage the shape $\Lambda$ peak arose at the place where the bottom of $\bigvee$ shape had occurred ( Fig 4, $\mathrm{R}_{8}$ of $\mathrm{C}_{1}$ and $\mathrm{R}_{3}$ of $\mathrm{C} 2$ ). The light intensity of $\Lambda$ stage was changing, sometimes more than one fold of the EB-CFB point and sometimes lower (Fig $4, \mathrm{R}_{4}-\mathrm{R}_{5}$ of $\mathrm{C}_{2}$ ), which indicated the sigle stripe was continuously altering. The intensity and position of stress folds on both sides of $\Lambda$ stage was changing continuously also (Fig $4, \mathrm{R}_{4}-\mathrm{R}_{6}$ of $\mathrm{C}_{2}$ ). In addition, dark stripes could also be seen on on both sides of $\Lambda$ s tage (Fig $4, R_{6}-R_{7}$ of $C_{2}$ ). When width of peak attained to 40-50 $\mu \mathrm{m}, \bigwedge$ stage changed to $\mathrm{M}$ stage.

c. $\mathrm{M}$ stage. The fluorescence intensity of both border lines of furrow changed greatly and quickly, and independent of each other (Fig $4, R_{1}-R_{5}, R_{4}-R_{3}$, and $R_{1}$ $\mathrm{R}_{3}$ in $\mathrm{C}_{3}-\mathrm{C}_{5}$ respectively). Sometimes the intensity of one border lines was 3 folds higher than that of EB-CFB point, while the other was not significant. The changes of fluorescence intensity and position of stress folds were also great. When the distanced between stress folds and border lines of furrow increased, the distance between two border lines also increase (Fig $4, \mathrm{R}_{2}-\mathrm{R}_{4}$ of $\mathrm{C}_{3}-\mathrm{C}_{4}$ ). It seemed as if stress folds were pulling the border lines outwards. When the distance between two border lines was approximate $100 \mu \mathrm{m}$, a fluorescence peak appeared between them, which was groove bottom, and the $\mathrm{M}$ stage turned to $\mathrm{W}$ stage.

d. W stage. The changes of border lines and stress folds of $\mathrm{W}$ stage were quite similar to that of the $\mathrm{M}$ stage. For example, when the distance between folds and border lines increased (Fig 4, $\mathrm{R}_{6}-\mathrm{R}_{9}$ of $\mathrm{C}_{3}-\mathrm{C}_{4}$ ), the distance between the two border lines also increased. When original folds were away for some distance from the border lines, new folds would appear between them (Fig 4, $\mathrm{R} 9$ of $\mathrm{C}_{4}$ ). It was plausible that folds were relaying the pulling of the border lines outward. Pulling the border line by stress folds was also indicated in Fig 3B where the stress folds were the longest and the nascent membrane was the broadest. The characteristics of $\mathrm{W}$ stage 
Movement of cleaving frog egg surface

C1

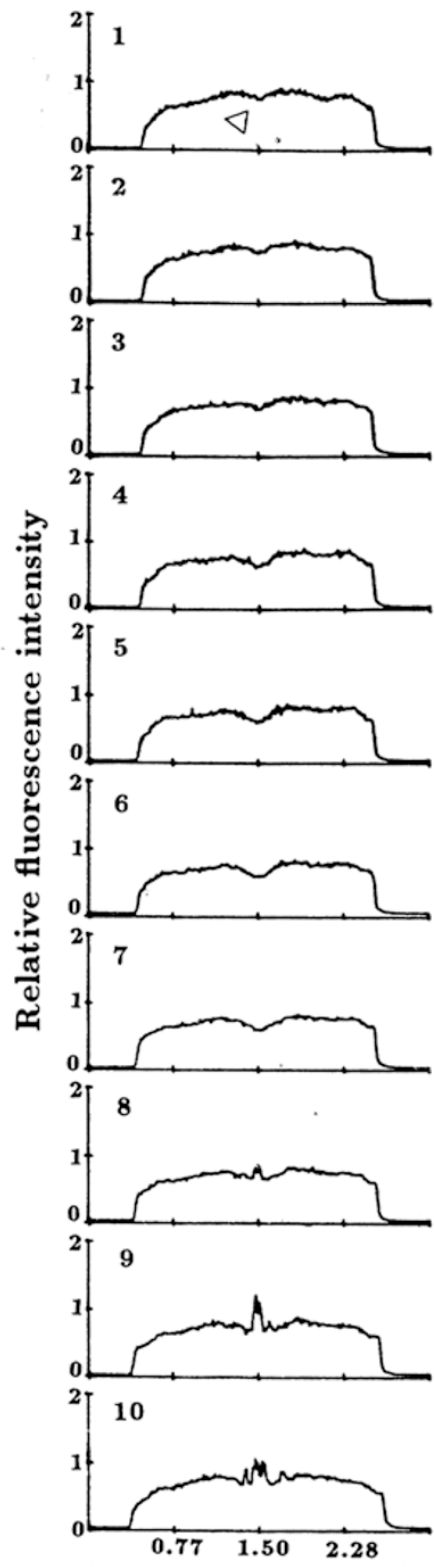

C2

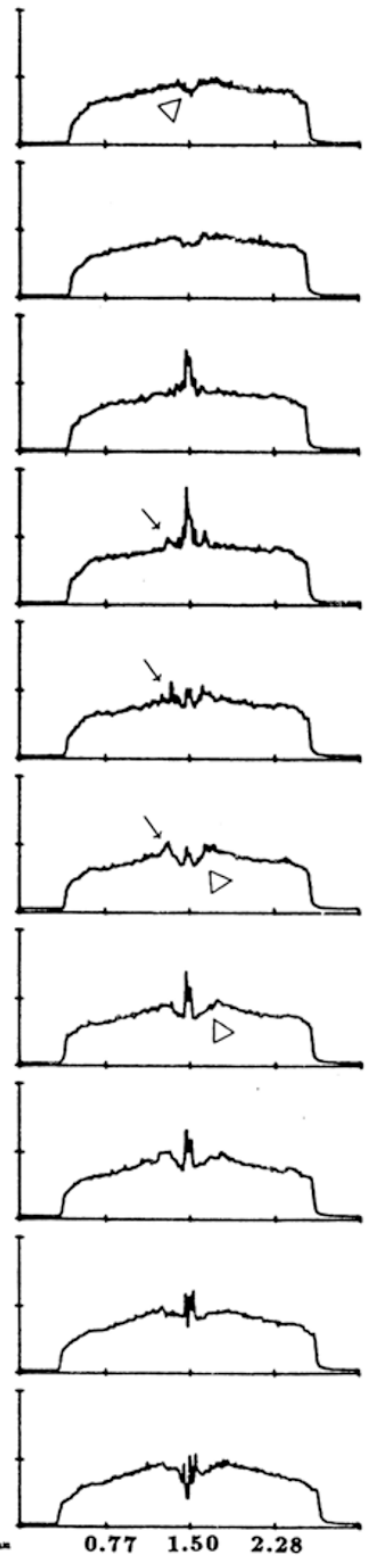

Fig 4 . The successive changes of surface fluorescence intensity of a cleaving egg. Scan was from left to right and from top to bottom. The first scan line was chosen at one end of cleavage furrow. Next columns were $100 \mu \mathrm{m}$ apart from the above towards another end of furrow. Time for scanning one row was $2 \mathrm{~min}$ and total time was $20 \mathrm{~min}$. 
$\mathrm{Ku} \mathrm{KY}$ et al.
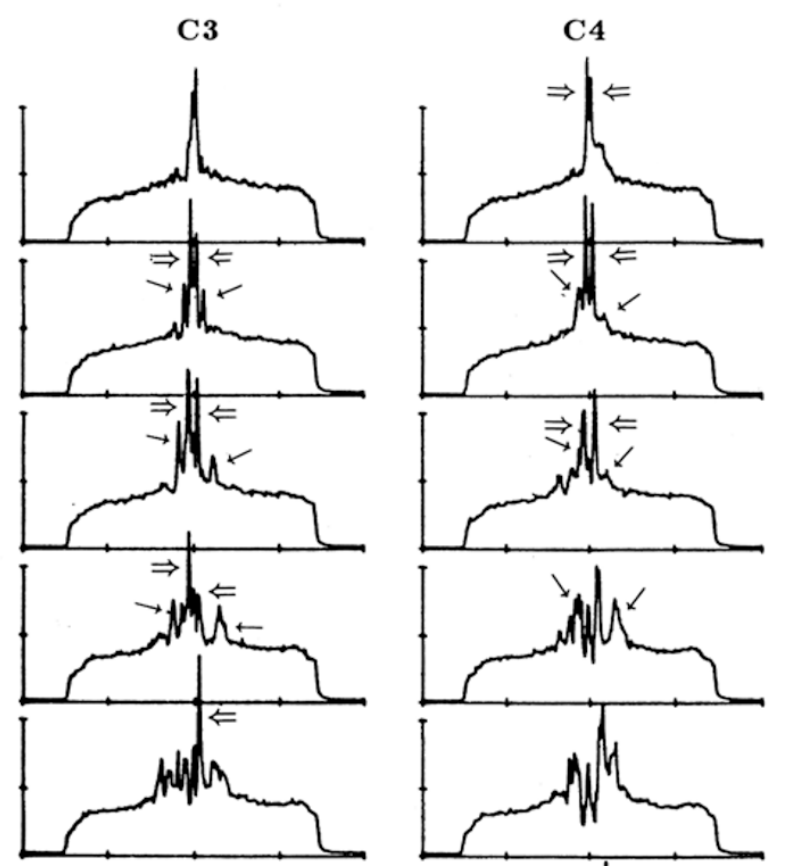

C5


Distance $(\mathbf{m m})$

$\triangle$, dark stipe. $\uparrow \uparrow$, fluorescence granules in nascent membrane. $\uparrow$, stress folds.

$\Uparrow$, border lines of cleavage furrow. 
was appearance of groove bottom which had uneven light intensity. At the beginning of appearance of groove bottom, its intensity was higher in nearly half of the observed eggs (8/18) than that of the EB-CFB point (Fig 4, $\mathrm{R}_{4}-\mathrm{R}_{7}$ in $\mathrm{C}_{4}$ ), which maintained for a fairly long time: 5 in 8 eggs over 4 min, 1 over 6 rain and 2 over 8 min. The intensity of the other 10 eggs, although lower than that of the EB-CFB point at the beginning, would arise quite soon. The intensity on nascent membrane all failed down rapidly. 2-4 min after the appearance of nascent membrane, its fluorescence falled down more than half, 6-8 min later, less than $1 / 4$ remained in most eggs. It indicated that nascent membrane expanded rapidly. The fluorescence intensity of nascent membrane was not even ( $\mathrm{Fig} 4, \mathrm{R}_{8}-\mathrm{R}_{10}$ in $\mathrm{C}_{4}$ ). This phenomenon was shown most clearly in Fig 3C. When the cleavage furrow went through the polar body extrusion point (its long axis was $59.3 \pm 6.3 \mu \mathrm{m}$ ) on old membrane, part of the polar body extrusion point o riginally located between border lines of M stage was pulled apart and many small pieces of old membrane were scattered on nascent membrane. This was consistent with the phenomenon that the residual fluorescence particles of old membrane remained between two border lines in early $\mathrm{W}$ stage (Fig 3B).

\section{B. The egg surface movement outsides of cleavage furrow}

On egg surface there were two kinds of movement, parallel or perpendicular to long axis of cleavage furrow. The parallel movement was shown by the appearance and disappearance of polar body extrusion point in the scanning curves of certain column and also in its appearance in next column (figure not shown). To calculate on the basis of polar body extrusion point being $60 \mu \mathrm{m}$, the maximum speed of parallel movement could exceed $10 \mu \mathrm{m} / \mathrm{min}$. Some might be $3-5 \mu \mathrm{m} / \mathrm{min}$ or even less, that meant the polar body extrusion point could be scanned on 10 curves in the same column i.e. in 20 rain. The perpendicular movement was shown in the increase of egg width. During $\mathrm{V}, \Lambda$ and $\mathrm{M}$ stages the width of eggs increased. The speed was about $10 \%$ of the egg diameter per min in general. During $\mathrm{W}$ stage, the change of width was small in its early appearance when the width of nascent membrane was substracted.

\section{DISCUSSION}

\section{Movement on egg surface}

\section{A. The formation of dark stripe}

Dark stripe was formed by the contraction of the presumptive single stripe either at the onset of cleavage or during the extension of furrow. By our result of short sight photogrammetry[11], the displacement measurement of the phytohemagglutinin-conjugated covaspheres binding to the corresponding receptors on egg surface of Rana amurensis, it was shown that 3-5 min before the appearance of a single stripe the 
egg surface surrounding the presumptive single stripe moved to the middle region which was going to form single stripe and the surrounding area forming dark stripe. During the extension of cleavage furrow the cytoplasm in front of single stripe could induce contraction $[12,13]$ and to pulling the surrounding egg surface inwards, on which the fluorescence intensity and protrusion density were decreased and those on the front of single stripe increased[6]. The above and the following phenomena indicated the mechanism of cleavage furrow extension was the same as that of the cleavage furrow formation.

\section{$B$. The unevenness of contraction of cleavage furrow bottom}

The contraction of cleavage furrow bottom was resulted from the contraction of actin in contractile arc[14]. Thus the originally more or less evenly distributed granular protrusions of egg surface turned out to be vesicular ones of $\mathrm{v}$ arying sizes and nascent membrane appeared among the clusters of vesicular protrusions (ref 15, Fig 13). This was consistent with the unevenness of fluorescence seen on groove bottom (ref 16, Fig 3). During laser scanning when light beam failed on the clustered vesicular protrusions, the fluorescence intensity significantly increased, while falled on nascent membrane the intensity significantly decreased. The unevenness of contraction of cleavage furrow bottom provided a morphological evidence for the contraction model of contractile ring as suggested by Satterwhite and Pollard[171.



Fig 5 A. Diagram of morphology and movement of cleaving egg surface

B. The enlargement of enclosed area in Fig 5A. Arrow denoting tension between border line and contractile arc. ds: dark stripe; cs: contractile arc; nam: nascent membrane; bl: border line; sf: stress fold. 
Movement of cleaving frog egg surface

\section{The contraction of border line of cleavage furrow}

Border line of cleavage furrow formed through the procession of further aggregation, contraction and differentiation of the protrusions on the borders of single stripe before it turned into tight junnction[18]. The contraction of border line would cause the furrow shortened and invaginated, and make stress folds conspicous.

\section{Egg surface movement outside of cleavage furrow}

The egg surface movement was different in direction and speed at different places[19$21]$. This may depend on the vector sum exerted on that place。

\section{The initiation of nascent mem brane formation}

Although many scientists believe that formation of nascent memrane is resulted from insertion of nascent membrane precursors, cytoplasmic vesicles and/ or molecules into old membrane[22-24], yet scarcely toned the problem: what initiates the insertion processes. More than a decade ago, Schroeder proposed imposed mechanical stress, such as stress generated by contractile arc, could induce such insertion [25]. However, from then on there have been few comments. In 1990, we proposed in the Fnist Congress of Ascian Pacific Organization of Cell Biology that the stretching of egg surface by the outsides of cleavage furrow, through such as stress folds, might be the factor to initiate nascent membrane formation[26]. One participant asked me whether I had done the experiment to make the egg into halves. My answer was "yes". VIk had done the experiment on Rana amurensis eggs using glass fiber to press the denuded egg to make it into two as Japanese scientists worked on newt eggs[27]. We had also done experiment on eggs of Rana amurensis using hatching enzyme to produce a hole at fertilization membrane to let egg surface and cytoplasm to flow out rapidly and the egg forming calabash shape[28] as Sawai using scissors to cut a slit on fertilization membrane of newt egg[29]. In all these experiments no nascent membrane had been found. It looked as if these experiments had denied the proposition of imposed mechanical stress. In fact it was not. Since on making the egg into two, the stress was acting on "whole" or a fairly large area of egg surface, such as around both sides of glass fiber, while during cleavage the stress produced by stress folds was only on the egg surface between tow border lines of cleavage furrow. The latter area was much smaller than the former. Therefore, in the latter cases the stress acted on unit area was much bigger. The experiments of artificial making the egg into two could only indicate the viscoelasticity of egg surface was big. During $\mathrm{M}$ stage. the distance between two border lines was initially about $50 \mu \mathrm{m}$ and increased to $100 \mu \mathrm{m}$ in the end, and no nascent membrane would appear (Fig $4 \mathrm{R}_{1}-\mathrm{R}_{4}, \mathrm{R}_{1}-\mathrm{R}_{3}$ and $\mathrm{R}_{1}-\mathrm{R}_{7}$ in $\mathrm{C}_{3}-\mathrm{C}_{5}$ respectively). During artificial making the egg into two, the surface only increased $1 / 3-1 / 4[19]$. Thus nascent membrane would not appear. Only when the stress exceeded certain threshold and making the movement of egg surface above certain extent, thus initiated the insertion of a sufficient large 
amount of vesicles and/ or molecules into old membrane and the nascent membrane could be seen only then.

Before introducing of evidence for stress as a factor to initiate nascent membrane formation,we should understand the role of stress fold acting as a sign of increasing the stress. Three indications were given below: (1) First, we thought that in natural condition egg cleaving in fertilization membrane, part of old membrane and all nascent membrane sinkinng into furrow were due to the contraction of contractile arc which pulled the nascent membrane and also through nascent membrane pulling part of the old membrane into furrow. Then, we put eggs enclosed in fertilization membrane into solution containing detergent, Brij, the area of nascent membrane increased[18] and nascent membrane exposed[23]. This indicated that the pulling force of contractile arc to border lines being decreased by the increasing area of nascent membrane and the pulling force around border lnes through stress folds diaplayed and part of the nascent membrane exposed out. (2) In Fig 3B where the stress folds were the longest which meant the pulling force was the strongest, and the area of nascent membrane became the broadest. (3) In 1991, Kobuta et al[30] found an af mutant of newt, its egg could cleave. During cleavage in fertilization membrane tress folds appeared and nascent membrane exposed, but there was no contractile arc. This indicated that stress through stress folds could initiate the formation of nascent membrane and to expose it. However, in the absence of contractile arc the cleavage furrow regressed finally. This implied that during normal cleavage stress fbld and contractile arc work independently and consequently.

The evidence for initiation of nascent membrane formation within cleavage furrow by stress through stress folds as shown by Kulota et al [30] and the evidence on uncleaved eggs by stress was given by us[31]. When FITC-stained uncleaved, naked eggs of Rana amurensis were put into solution containing 0.75-1.5 $\mu \mathrm{mole} / \mathrm{L}$ polylysine at $\mathrm{pH} 7.0-7.2$, the cation groups of polylysine bound to anion groups on egg surface. Thus produced the transmembrane effect, which caused consistent cont: raction of preexisting membrane and numerous bright pinpoints appeared on the originally evenly distributed fluorescein stained egg surface, and these pinpoints further aggregated into protruded patches formed by a large amount of preexisting membrane and melannin granules (TEM observation, Figure unpublished.). Thus the stress among pinpoints and patches was increased consistently. When the stress exceeded certain threshold, nascent membrane without protrusion and pigment appeared among the patches and expanded as the patches continuously gathered to animal pole until the vegetative hemisphere of egg broken. If the polylysine solution was $0.3 \mu \mathrm{mole} / \mathrm{L}$ or less, bright pinpoints appeared but not further aggregated, nascent membrane did not appear. This indicated that when the stress pulled preexisting membranes apart not large enough nascent membrane could not the be initiated. If the stress pulling preexisting membrane enough apart could actually initiate nascent membrane formation, then pulling preexisting membrane near both sides of border lines toward each other would decrease the area of nascent membrane and change the cleavage 
furrow shape. This inference was proved to be true by works on eggs of Xenopus[32] and Rana amureniss[16,18,33]. While eggs were put in solution containing soybean or wheat germ agglutinins, nascent membrane area whithin furrows was decreased [18], and part of the originally exposed nascent membrane was sheltered, so cleavage furrow changed to dumb-bell shape[16] or even only a trace of cleavage furrow but no nascent membrane within could be seen, so cleavage furrow changed to streak shape[18,32,33]. These might be due to that after lectins bound to their corresponding receptors which were scarce on nascent membrane but numerous on preexisting membrane[15], the cytoskeleton under preexisting membrans was effected through transmembrane effect[32], the net result was pulling the preexisting membrane lying both sides of furrow toward each other, thus the cleavage furrow was narrowed. If the lectins were heavily bound to their receptors and formed lattice on egg surface, it decreased or prevented the contraction produced by imposed stress. Thus, only the trace of cleavage furrow could be seen[32,33]. The degree of decrease, shelter or arrest of nascent membrane depended on the concentration of lectins used and the time of exposure to lectins solution. Furthermore, the effect of lectins could be abolished if the lectins were washed out or their competitive inhibitors were added. From above, it is evident that not only the initiation of nascent membrane formation but also the cleavage furrow shape of denuded egg and the size of nascent membrane are dependent on local surface stress.

In summary, from biomechanical point of view, cytokinesis is a process of several kinds of consecutive cell surface movement, including (1) contraction of furrow tip which results in the appearance of dark stripe, stress folds and extension of cleavage furrow (arrow inward in Fig 5A), (2) temporary contraction of border line which makes egg surface invaginating and stress folds more conspicuous (Fig 5B), (3) stretch of preexisting membrane near border line (arrow outward in Fig $5 \mathrm{~A}$ ) which increases the tension between border lines leading to initiation of hastent membran formation and exposure of nascent membrane and (4) persistent contraction of conttactile arc which results in the enlargement of nascent membrane and invagination of furrow bottom. The extent of each of these four kinds of movement is different with different eggs and different features of cleavage furrow appear.

\section{REFERENCES}

[1] Dan K, Kojima MK. A study on the mechanism of cleavage in the amphibian egg. J Exp Biol 1963; 40:7-14.

[2] Sawai T. On propagation of cortical factor and cytoplasmic factor participating in cleavage furrow formation of the newt's egg. Dev. Growth Differ 1980; 22:437-44.

[3] Mabuchi I, Tsukita Sh, Tsukita Sa, Sawai T. Cleavage furrow isolated from newt eggs: Contraction, organization of the actin filaments, and protein components of the furrow. Proc Natl Acad Sci USA 1988; 85:5966-70.

[4] Sato N, Yonemurea S, Obinata T, Tsukita Sa, Tsukita Sh. Radixin, a barbed end-capping 
actin-modulating protein, is concentrated at the cleavage furrow during cytokinesis. J Cell Biol 1991; 113:321-30.

[5] Yonemura S, Nagafuchi A, Sato N, Tsukita S. Concentration of an integral membrane protein, CD43 (Leukosialin, Sialophorin), in the cleavage furrow through the interaction of its cytoplasmic domain with actin-based cytoskeletons. J Cell Biol 1993; 120:437-49.

[6] Ku KY, Xu CT, Zhang KH. Cleavage furrow formation of Rana Amurensis eggs observed with fluorescence pattern photobleaching. Cell Biol Inter Rep 1988; 12:175-87.

[7] Bluemink JK. Cytokinesis and cytochalasin-induced furrow regression in the first cleavage zygote of Xenopus laevis. Z Zellforsch Mikrosk Anat 1971; 121:102-26.

[8] Singal PK, Sanders EJ. An ultrastructural study of the first cleavage of Xenopus embryos. J Ultrastruct Res 1974; 47: 433-51.

[9] Akkas N. Editor of "Biomechanics of Cell Division” . Plenum Press. New York and London 1987.

[10] White JG, Borisy GG. On the mechanisms of cytokinesis in animal cell. J Theor Biol 1983; 101:289-316.

[11] Gao QR, Liu XH, Zhang KH, et a1. Surface contraction wave and cleavage initiation contraction on the fertilized egg of Rana Amurensis. Acta Biol Exp Sinica 1993; 26: 449-61.

[12] Sawai T. Cytoplasmic and cortical factors participating in cleavage furrow formation in eggs of three amphibian genera: Ambystoma, Xenopus and Cynops. J Embryol Exp Morph 1983; 77:243-54.

[13] Jiang $\mathrm{S}, \mathrm{Li} \mathrm{MF}, \mathrm{Ku} \mathrm{KY}$. Bioassay and isolation of contraction factor(s) from Rana Amurensis embryo. Acta Biol Exp Sinica 1988; 21:383-91.

[14] Schroeder TE. The contractile ring and furrowing in dividing cells. Annals New York Academy of Sciences 1990; 582:78-87.

[15] Hong LS, Gao KG, Cheng PR, Ku KY, A scanning electron microscopic study of the formation and regression of the first cleavage furrow of Rana Amurensis. Acta Biol Exp Sinica 1981; 14:161-71.

[16] Ku KY, Hong LS, Jiang S. 1982. Morphological changes of the surface of the eggs of Rana Amurensis during first cleavage by fluorescence microscopy. Acta Biol Exp Sinica 15: 375-85.

[17] Satterwhite LL, Pollard TD. Cytokinesis. Current Opinion in Cell Biology. 1992; 4:43-52.

[18] Gao QR, Zhang KH, Hong LS, Xu CT, Gu Guo-yan (formerly Ku KY). The development of the region between the preexisting and nascent membranes during the first cleavage of Rana Amurensis eggs. Cell Reseach 1992; 2:35-43.

[19] Selman GG, Waddington CH. The mechanism of cell division in the cleavage of the newt's egg. J Exp Biol 1955; 32:700-35.

[20] Byers TJ, Armstron PB. Membrane protein redistribution during Xenopus first cleavage. J Cell Biol 1986; 102: 2176-84.

[21] Sawai T. Movement of the cell surface and change in surface area during cleavage in the newt' $\mathrm{s}$ egg. J Cell Sci 1976; 21:537-51.

[22] Bluemink JK, De Laat SW. New membrane fclrmation during cytokinesis in normal and cytochalasin B-treated eggs of Xenopus laevis. I. Electron microscope observations. J Cell Biol 1973; 59:89-108.

[23] Gao QR, Hong LS, Jiang S, Ku KY. Detergent, Brij, increasing the area of new surface membrane during the early cleavage of eggs of Rana Amurensis. Cell Biol Inter Rep 1986; 10:969-77.

[24] Bieliavsky N, Geuskeas M. Interblastomeric plasma membrane formation during cleavage of Xenopus laevis embryos. J Submicrosc Cytol Pathol, 1990; 22:445-57.

[25] Schreder TE. Interrelations between the cell surface and the cytoskeleton in cleaving sea uvclin eggs. In: cytoskeletal elements and plasms membrane organization ( poste G. \& Nicolzon,G. L. eds). 1981:pp 169-215.

[26] Ku KY, Xu CT, Zhang KH, Gao QR, Jiang S. The Movement of egg surface of Rana Amurensis before and during cytokinesis. First Congress of Asian-pacific Organization for Cell Biology. 


\section{Movement of cleaving frog egg surface}

Abstracts. 1990: s5.5, pp. 67-8.

[27] Kobayakawa Y, Kubota HY. Temporal pattern of cleavage and the onset of gastrulation in amphibian embryos developed from with the reduced cytoplasm. J Embryol exp Morph 1981; 62:83-94.

[28] Li MF, Hong LS, Ku KY . A rapid method for the removal of jelly coat and vitelline membrane of fertilized eggs of Rana Amurensis. Acta Biol Exp Sinica 1980; 13:105-11.

[29] Sawai T. Cychc changes in the cortical layer of non-nucleated framents of the newt's egg. J Embryol Exp Morph 1979; 51:183-93.

[30] Kubota HY, Itoh K, Kubota MA. Cytological and maternal-effect mutant embryos with abnormal cleavage furrow formation in Xenopus laevis. Dev Biol 1991; 144:145-51.

[31] Wu YL, Jiang $\mathrm{S}, \mathrm{Ku} \mathrm{KY}$. The effects of polylysine on the aggregation of surface and on the cleavage furrow of Rana Amurensis eggs. Acta Biol Exp Sinica 1993; 26:51-64.

[32] Tencer R. Transmembrane effects of lectins. I. Effects of wheat germ agglutinin and soybean agglutinin on furrow formation and cortical wound healing in Xenopus laevis eggs. Exp Cell Res 1978; 116: 253-60.

[33] Ku KY, Hong LS. The inhibitory effects of lectins on the exposure of new membrane during the first cleavage of Rana Amurensis eggs. Acta Biol Exp Sinica 1984; 17: 329-35.

Received 27-10-1994. Revised 6-4-1995. Accepted 25-4-1995. 